\title{
Entropy Generation due to MHD Mixed Convection of Nanofluid in a Vertical Channel with Joule Heating and Radiation effects
}

\author{
D. Srinivasacharya ${ }^{\# 1}, \mathrm{Md}$. Shafeeurrahman ${ }^{\# 2}$ \\ \# Department of Mathematics, National Institute of Technology, \\ Warangal-506004, India \\ 11dsc@nitw.ac.in; dsrinivasacharya@yahoo.com \\ ${ }^{2}$ second.author@second.com
}

\begin{abstract}
In this article, the effects of Joule heating and radiation on entropy generation due to laminar mixed convective flow of an incompressible electrically conducting nanofluid in vertical channel is studied. The governing equations are non-dimensionalized and then solved by using homotopy analysis method. The effects of magnetic, Joule heating, Brinkman number and radiation on the velocity in flow direction, temperature, nanoparticle concentration, Bejan number and entropy generation are analyzed and presented geometrically.
\end{abstract}

Keyword - Entropy generation, Mixed convection, Radiation, Joule heating, MHD, Nanofluid, Vertical channel

\section{INTRODUCTION}

The study of convective heat transfer in nanofluids has received considerable theoretical and practical interest in view of its use in a wide variety of engineering applications. These applications include cooling of nuclear reactor, power generating systems, automobile engines, welding equipment, heat exchanging in electronics devices and computers. Nanofluids are prepared by the uniform dispersion and suspension of nanometer sized metallic particle into convective heat transfer fluids such as water, oil, or ethylene glycol [1]. Choi [1] verified that the nanofluids have more thermal-conductivity proportionate to the base fluids. Mixed convection heat transfer and nanofluid flow in a vertical channel, a frequently encountered geometry in thermal engineering equipment, has been concentrating on vast investigation for many years.

Number of studies were conducted in recent years, on vertical channel filled with nanofluid by considering distinct types of convectional base fluids with particular nanoparticles. Hang and Pop [2] verified the mixed convective nanofluids flow in a vertical channel. Dehkordi and Hajipour [3] analyzed the mixed convective heat transfer of nanofluid flow between parallel plates. Grosan and Pop [4] analyzed the fully developed and mixed convective nanofluid flow in a vertical channel. Hang et al [5] studied the mixed convective nanofluid flow in a vertical channel using the Buongiorno method. Nield and Kuznetsov [6] discussed the forced convectional nanofluid flow in a channel. Fakour and Vahabzadeh [7] studied the review of mixed convective flow of a nanofluid in a vertical channel. Makhataret et al. [8] analyzes flow reversal of mixed convective flow of nanofluids with porous medium in a vertical channel. Das et al. [9] analyzed the nanoparticle volume fraction on mixed convective nanofluid flow in a vertical plates.

Several authors studied the effect of radiation parameter on convective flow of nanofluid together with heat and mass transfer from bodies of different geometries under various physical conditions due to its vast range of applications involving high temperatures such as gas turbines missiles, satellites, nuclear power plant, aircraft and space vehicles etc. Chamkha et al. [10] numerically studied the effects of thermal radiation on mixed convective flow around a cone inscribed in a porous medium filled with a nanofluid. Sheikholeslami and Davood [11] investigated the effect of thermal radiation and magnetic field on the unsteady flow of a nanofluid flow and heat transfer. On the other hand numerous studies have been carried out on the effect of Joule heating on fluid flow and heat transfer at various conditions and found that it plays notable effect on MHD flow and heat transfer. Anand et al. [12] analyzed the thermal radiation and Joule heating effects on MHD flow of a nanofluid in boundary layer. Srinivasacharya and Vijay [13] described the mixed convective flow of a nanofluid in a non-Darcy porous medium considering radiation effect. Hayat et al. [14] presented a model for Joule heating and solar radiation in MHD convection flow of nanofluid. 
The study of entropy generation, the amount of irreversibility associated with the real processes, has been growing interest as it is a powerful and useful optimization tool for a high range of thermal applications. The entropy generation destroys the system energy. Hence, the performance of the system can be improved by decreasing the entropy generation. Bejan $[15,16]$ introduced the entropy generation minimization method and developed its applications in engineering sciences. Since then several researchers have been studying the entropy generation analysis for different types of geometries with diverse fluids. Omid et al. [17] analyzed the significance of radiation parameter effect on entropy generation within nanofluids. Dehsara et al. [18] numerically analyzed the entropy generation in nanofluid flow in presence of variable magnetic field, viscous dissipation and solar radiation. Govindaraju et al. [19] investigated the entropy generation in MHD flow of a nanofluid. Rashidi et al. [20] presented the influence of thermal radiation on entropy generation in MHD blood flow of a nanofluid.

The survey of the literature reveals that the interaction of Joule heating and radiation effects with nanoparticles in a mixed convectional heat transfer flow in a vertical channel occupied by nanofluid has not been considered. Hence, the main aim of this article is to investigate the mixed convective flow of a nanofluid passing through a vertical channel with Joule heating and thermal radiation effect. The homotopy analysis method is used to solve the nonlinear differential equations. HAM [21] was first introduced by Liao, which is one of the most powerful technique to find the solution of strongly nonlinear equations. The effect of radiation, Joule heating and magnetic parameter on the velocity along the fluid direction, temperature, nanoparticle concentration, Bejan number and entropy generation is investigated..

\section{Problem formulation}

Consider a steady, laminar incompressible electrically conducting nanofluid flow through a vertical channel of width $2 d$. The $\mathrm{x}$-axis is taken to be in vertically upward direction of the flow through the central line of the channel and $y$-axis in the orthogonal direction to the flow as shown in figure 1. The plate $y=-d$ is maintained at temperature $T_{1}$ and nanoparticle volume fraction $\phi_{1}$, whereas the plate $y=d$ is maintained at $T_{2}$ and $\phi_{2}$ respectively. Rate of flow through the plates is assumed to be constant $v_{0}$ and uniformly equal for both the plates. A uniform magnetic field $B_{0}$ is taken in $y$-direction. A uniform pressure gradient in x-direction and buoyancy forces caused the mixed convective flow. The induced magnetic field is ignored as the magnetic Reynolds number is very low and all the fluid properties are considered as constant apart from the density in the buoyancy term. The fluid is considered to be a gray, absorbing/emitting radiation, but non-scattering medium. The Rosseland approximation [22] is used to describe the radiative heat flux in the energy equation. $u$ is the velocity along $x$ - direction, $T$ denotes the temperature and $\phi$ denotes the nanoparticle concentration.

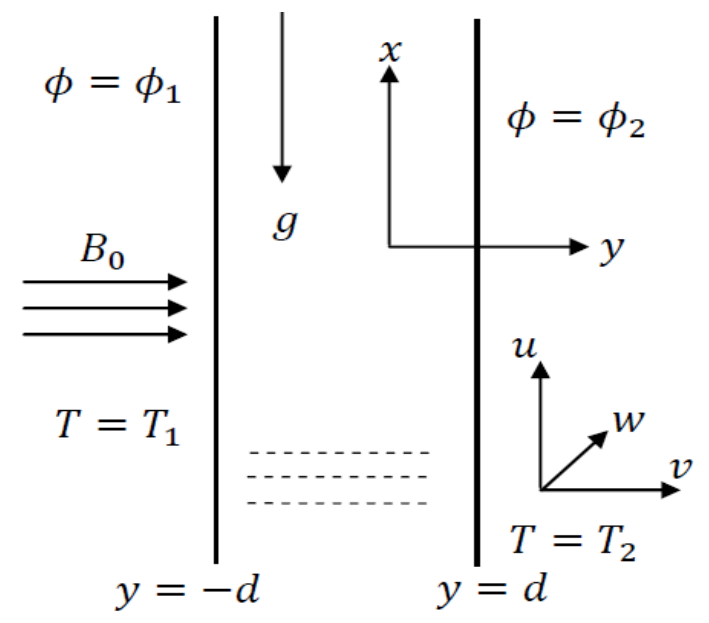

Figure 1: Geometry of the problem

Under these assumptions, the governing equations of the flow by considering the nanofluid model proposed by Buongiorno [23] are as follows:

$$
\begin{gathered}
\frac{\partial v}{\partial y}=0 \\
\rho_{f} v \frac{\partial u}{\partial y}=-\frac{\partial p}{\partial x}+\mu \frac{\partial^{2} u}{\partial y^{2}}+\left(1-\phi_{m}\right) \rho_{f_{0}} g \beta_{T}\left(T-T_{1}\right)-\left(\rho_{p}-\rho_{f_{0}}\right) g\left(\phi-\phi_{1}\right)-\sigma B_{0}^{2} u
\end{gathered}
$$




$$
\begin{gathered}
v \frac{\partial T}{\partial y}=\alpha \frac{\partial^{2} T}{\partial y^{2}}+\frac{2 \mu}{\rho c_{p}}\left(\frac{\partial u}{\partial y}\right)^{2}-\frac{16 T_{m}^{3} \sigma}{3 K_{f} \chi \rho c_{p}} \frac{\partial^{2} T}{\partial y^{2}}+\tau\left[D_{B} \frac{\partial T}{\partial y} \frac{\partial \phi}{\partial y}+\frac{D_{T}}{T_{m}}\left(\frac{\partial T}{\partial y}\right)^{2}\right]+\frac{1}{\rho c_{p}} \sigma B_{0}^{2} u^{2} \\
\frac{\partial \phi}{\partial y} v=\frac{D_{T}}{T_{m}} \frac{\partial^{2} T}{\partial y^{2}}+D_{B} \frac{\partial^{2} \phi}{\partial y^{2}}
\end{gathered}
$$

where the density is $\rho$, the pressure is $\mathrm{p}$, the electrical conductivity is $\sigma$, Brownian diffusion coefficient is $D_{B}$, the specific heat capacity is $C p$, the viscosity coefficient is $\mu$, the coefficients of thermal expansion is $\beta_{T}$, the effective thermal diffusivity is $\alpha$, the acceleration due to gravity is $g$, the thermophoretic diffusion coefficient is $D_{T}$, Stefan-Boltzman constant is $\sigma_{1}$ and coefficient of mean absorption is $\chi$.

The conditions on the boundary are:

$$
\begin{aligned}
& u=0, \quad v=v_{0}, \quad T=T_{1}, \quad \phi=\phi_{1} \quad \text { on } \quad y=-d, \\
& u=0, \quad v=v_{0}, \quad T=T_{2}, \quad \phi=\phi_{2} \quad \text { on } \quad y=d,
\end{aligned}
$$

Introducing the following non-dimensional variables

$$
\eta=\frac{y}{d}, f=\frac{u}{u_{0}}, p=\frac{\mu u_{0}}{d^{2}} P, \theta=\frac{T-T_{1}}{T_{2}-T_{1}}, S=\frac{\phi-\phi_{1}}{\phi_{2}-\phi_{1}},
$$

in equations (1) - (5), we get the nonlinear differential equations as

$$
\begin{gathered}
f^{\prime \prime}-R f^{\prime}+\frac{G r}{R e}(\theta-N r S)+M^{2} f-A=0 \\
\left(1+\frac{4}{3} R d\right) \theta^{\prime \prime}-R \operatorname{Pr} \theta^{\prime}+\operatorname{Pr} N b \theta^{\prime} S^{\prime}+\operatorname{Pr} N t \theta^{\prime 2}+2 B r\left(f^{\prime}\right)^{2}+J f^{2}=0 \\
S^{\prime \prime}-R L e S^{\prime}+\frac{N t}{N b} \theta^{\prime \prime}=0
\end{gathered}
$$

where the kinematic viscosity coefficient is $v, \operatorname{Pr}=\mu C_{P} / k_{f}$ represents the Prandtl number and $L e=v / D_{B}$ represents the Lewis number, $R=v_{0} d / v$ is the suction/injection parameter, $G r=(1-\phi) g \beta_{T}\left(T_{2}-T_{1}\right) d^{3} / v^{2}$ is the Grashof number, $R e=u_{0} d / v$ is the Reynold's number, $M^{2}=B_{0}{ }^{2} d^{2} \sigma / \mu$ is the magnetic parameter, $A=\left(d^{2} / \mu u_{0}\right) \partial p / \partial x$ is a constant pressure gradient, $B r=\mu u_{0}^{2} /\left(k_{f}\left(T_{2}-T_{1}\right)\right)$ represents the Brinkman number, the Brownian motion parameter is $N b=\tau D_{B}\left(\phi_{2}-\phi_{1}\right) / v, N t=\tau D_{T}\left(T_{2}-T_{1}\right) /\left(T_{m} v\right)$ is the thermoporesis parameter and buoyancy ratio is $N r=\frac{\left(\rho_{p}-\rho_{f_{0}}\right)\left(\phi_{2}-\phi_{1}\right)}{\rho_{f_{0}} \beta_{T}\left(T_{2}-T_{1}\right)\left(1-\phi_{m}\right)}$, Joule heating parameter is $J=\frac{u_{0}^{2} \sigma B_{0}^{2} d^{2}}{\left(T_{2}-T_{1}\right) k_{f}}$ and $R d=\frac{4 \sigma_{1}}{3 \chi} \frac{T_{m}^{3}}{K_{f}}$ is the Radiation parameter.

The corresponding conditions (5) on boundary are

$$
\begin{aligned}
& S=0, \quad \theta=0, \quad f=0 \quad \text { at } \quad \eta=-1, \\
& S=1, \quad \theta=1, \quad f=0 \quad \text { at } \quad \eta=1 .
\end{aligned}
$$

\section{III.HOMOTOPY SOLUTION}

To obtain the HAM solution (For more details on homotopy analysis method see the works of Liao [21, 24-26] ), first we guess the initial values as

$$
f_{0}(\eta)=0, \quad \theta_{0}(\eta)=\frac{\eta}{2}+\frac{1}{2}, \quad \text { and } \quad S_{0}(\eta)=\frac{\eta}{2}+\frac{1}{2} .
$$

and the auxiliary linear operators as $L_{i}=\partial^{2} / \partial \mathrm{y}^{2}$, for $i=1,2,3$ such that $L_{1}\left(c_{1}+c_{2} y\right)=0, L_{2}\left(c_{3}+c_{4} y\right)=0$ and $L_{3}\left(c_{5}\right.$ $\left.+c_{6} y\right)=0$, where $c_{i},(i=1,2, \ldots 6)$ are constants.

The zero $^{\text {th }}$ order deformation, which is given by

$$
\begin{aligned}
& (1-p) L_{1}\left[f(\eta ; p)-f_{0}(\eta)\right]=p h_{1} N_{1}[f(\eta ; p)], \\
& (1-p) L_{2}\left[\theta(\eta ; p)-\theta_{0}(\eta)\right]=p h_{2} N_{2}[\theta(\eta ; p)], \\
& (1-p) L_{3}\left[S(\eta ; p)-S_{0}(\eta)\right]=p h_{3} N_{3}[S(\eta ; p)] .
\end{aligned}
$$


where

$$
\begin{aligned}
& N_{1}[f(\eta ; p), \theta(\eta ; p), S(\eta ; p)]=f^{\prime \prime}-R f^{\prime}+\frac{G r}{R e}(\theta-N r S)-M^{2} f-A, \\
& N_{2}[f(\eta ; p), \theta(\eta ; p), S(\eta ; p)]=\left(1+\frac{4}{3} R d\right) \theta^{\prime \prime}-R \operatorname{Pr} \theta^{\prime}+\operatorname{Pr} N b \theta^{\prime} S^{\prime}+\operatorname{Pr} N t \theta^{\prime 2}+2 B_{r}\left(f^{\prime}\right)^{2}+J f^{2}, \\
& N_{3}[f(\eta ; p), \theta(\eta ; p), S(\eta ; p)]=S^{\prime \prime}-R L e S^{\prime}+\frac{N t}{N b} \theta^{\prime \prime} .
\end{aligned}
$$

Here $\mathrm{p} \in[0,1]$ is the embedded parameter and $h_{i},(i=1,2,3$.) are auxiliary parameters.

From $\mathrm{p}=0$ to $\mathrm{p}=1$, we can have

$$
\begin{aligned}
& f(\eta ; 0)=f_{0}, S(\eta ; 0)=S_{0}, \theta(\eta ; 0)=\theta_{0}, \\
& f(\eta ; 1)=f(\eta), S(\eta ; 1)=S(\eta), \theta(\eta ; 1)=\theta(\eta) .
\end{aligned}
$$

Thus, as $\mathrm{p}$ varying from initial value 0 to final value $1, f, \theta$ and $S$ changes from $f_{0}, \theta_{0}$ and $S_{0}$ to the final solution $f(\eta), \theta(\eta)$ and $S(\eta)$. Using Taylor's series one can write

$$
\begin{aligned}
& f(\eta ; p)=f_{0}+\sum_{m=1}^{\infty} f_{m}(\eta) p^{m}, \quad f_{m}(\eta)=\left.\frac{1}{m !} \frac{\partial^{m} f(\eta ; p)}{\partial p^{m}}\right|_{p=0}, \\
& \theta(\eta ; p)=\theta_{0}+\sum_{m=1}^{\infty} \theta_{m}(\eta) p^{m}, \quad \theta_{m}(\eta)=\left.\frac{1}{m !} \frac{\partial^{m} \theta(\eta ; p)}{\partial p^{m}}\right|_{p=0}, \\
& S(\eta ; p)=S_{0}+\sum_{m=1}^{\infty} S_{m}(\eta) p^{m}, \quad S_{m}(\eta)=\left.\frac{1}{m !} \frac{\partial^{m} S(\eta ; p)}{\partial p^{m}}\right|_{p=0} .
\end{aligned}
$$

and choose the values of the auxiliary parameters for which the series (15) are convergent at $\mathrm{p}=1$ i.e.,

$$
f(\eta)=f_{0}+\sum_{m=1}^{\infty} f_{m}(\eta), \theta(\eta)=\theta_{0}+\sum_{m=1}^{\infty} \theta_{m}(\eta), S(\eta)=S_{0}+\sum_{m=1}^{\infty} S_{m}(\eta)
$$

is convergent.

The equivalent boundary conditions are

$$
S(-1, p)=0, S(1, p)=1, \theta(-1, p)=0, \theta(1, p)=1, f(-1, p)=0, f(1, p)=0 .
$$

The $\mathrm{m}^{\text {th }}$-order deformation is given by

$$
\begin{aligned}
& L_{1}\left[f_{m}(\eta)-\chi_{m} f_{m-1}(\eta)\right]=h_{1} R_{m}^{f}(\eta), \\
& L_{2}\left[\theta_{m}(\eta)-\chi_{m} \theta_{m-1}(\eta)\right]=h_{2} R_{m}^{\theta}(\eta), \\
& L_{3}\left[S_{m}(\eta)-\chi_{m} S_{m-1}(\eta)\right]=h_{3} R_{m}^{S}(\eta) .
\end{aligned}
$$

where

$$
\begin{aligned}
& R_{m}^{f}(\eta)=f^{\prime \prime}-R f^{\prime}+\frac{G r}{R e}(\theta-N r S)-M^{2} f-\left(1-\chi_{m}\right) A \\
& \begin{aligned}
R_{m}^{\theta}(\eta)=\left(1+\frac{4}{3} R d\right) \theta^{\prime \prime}-\operatorname{RPr} \theta^{\prime}+\operatorname{PrNb} \sum_{n=0}^{m-1} \theta_{m-1-n}^{\prime} S_{n}^{\prime}+\operatorname{PrNt} \sum_{n=0}^{m-1} \theta_{m-1-n}^{\prime} \theta_{n}^{\prime} \\
+2 B r \sum_{n=0}^{m-1} f_{m-1-n}^{\prime} f_{n}^{\prime}+J \sum_{n=0}^{m-1} f_{m-1-n} f_{n}
\end{aligned} \\
& R_{m}^{S}(\eta)=S^{\prime \prime}-R L e S^{\prime}+\frac{N t}{N b} \theta^{\prime \prime}
\end{aligned}
$$

for an integer $m$

$$
\chi_{m}=0 \text { for } m \leq 1
$$

$=1$ for $m>1$ 


\section{IV.CONVERGENCE}

In HAM, it is essential to see that the series solution converges. Also, the rate of convergence of approximation for the HAM solution mainly depend on the values of $h$. To find the admissible space of the auxiliary parameters, $h$-curves are drown for $20^{\text {th }}$-level of approximation and shown in figure 2 . It is oserved from this figure that the permissible range for $h_{1}, h_{2}$ and $h_{3}$ is $-0.6<h_{1}<-0,-0.7<h_{2}<-0$ and $-1.0<h_{3}<-0.2$, respectively.

In order to obtain the optimal values of the auxiliary parameters, the following average residual errors [25] are computed and found that these errors are least at $\mathrm{h}_{1}=-0.3, \mathrm{~h}_{2}=-0.64$ and $\mathrm{h}_{3}=-0.73$.

$$
\begin{aligned}
& E_{f, m}=\frac{1}{2 k} \sum_{i=-k}^{k}\left(N_{1}\left[\sum_{j=0}^{m} f_{j}(i \Delta t)\right]\right)^{2}, E_{\theta, m}=\frac{1}{2 k} \sum_{i=-k}^{k}\left(N_{2}\left[\sum_{j=0}^{m} \theta_{j}(i \Delta t)\right]\right)^{2}, \\
& E_{S, m}=\frac{1}{2 k} \sum_{i=-k}^{k}\left(N_{3}\left[\sum_{j=0}^{m} S_{j}(i \Delta t)\right]\right)^{2} .
\end{aligned}
$$

where $\Delta t=1 / k$ and $\mathrm{k}=5$. Also, for different values of $m$ the series solutions are calculated and noticed that the series (16) converges in the total area of $\eta$. Further, the graphs of the ratio

$$
\beta_{f}=\left|\frac{f_{m}(h)}{f_{m-1}(h)}\right|, \quad \beta_{\theta}=\left|\frac{\theta_{m}(h)}{\theta_{m-1}(h)}\right|, \quad \beta_{S}=\left|\frac{S_{m}(h)}{S_{m-1}(h)}\right| .
$$

in contrast to the number of terms $m$ in the homotopy series are calculated and observed that the series (16) converges to the exact solution.

\section{ENTROPY GENERATION}

The volumetric rate of local entropy generation of a nanofluid in vertical channel can be expressed as

$$
S_{G}=\frac{K_{f}}{T_{1}^{2}}\left[\left(\frac{\partial T}{\partial y}\right)^{2}+\frac{16 \sigma}{3 \chi} \frac{T_{m}^{3}}{K_{f}}\left(\frac{\partial T}{\partial y}\right)^{2}\right]+\frac{\sigma B_{0}^{2} u^{2}}{T_{1}}+\frac{2 \mu}{T_{1}}\left(\frac{\partial u}{\partial y}\right)^{2}+\frac{R D}{\phi_{1}}\left(\frac{\partial \phi}{\partial y}\right)^{2}+\frac{R D}{T_{1}}\left(\frac{\partial T}{\partial y}\right) \cdot\left(\frac{\partial \phi}{\partial y}\right)
$$

where $R$ is the universal gas constant and $D$ is the species diffusivity through the fluid.

The entropy generation number $N s$ is the ratio of the volumetric entropy generation rate to the characteristic entropy generation rate according to Bejan[16]. Therefore $N s$ is given by

$$
N s=\left(1+\frac{4}{3} R d\right) \theta^{\prime 2}+\frac{1}{\Omega_{1}}\left(2 B_{r} f^{\prime 2}+J f^{2}\right)+\phi_{2} S^{\prime 2}+\phi_{3} \theta^{\prime} S^{\prime}
$$

The dimensionless coefficients are $\phi_{2}$ and $\phi_{3}$, called irreversibility distribution ratios which are related to diffusive irreversibility,

given by

$$
\phi_{2}=\frac{R D}{K_{f} \cdot \Omega_{1}}\left(\frac{\Omega}{\Omega_{1}}\right) \Delta \phi \quad \phi_{3}=\frac{R D}{K_{f} \cdot \Omega_{1}} \cdot \Delta \phi
$$

where $\Omega=\frac{\Delta \phi}{\phi_{1}}, \Omega_{1}=\frac{\Delta T}{T_{1}}$ are the concentration and temperature ratios, respectively and $\frac{K_{f}(\Delta T)^{2}}{d^{2} T_{1}^{2}}$ is the characteristic entropy generation rate. The Eq.(23)can be expressed as

$$
N s=N h+N v
$$

The entropy generation due to heat transfer irreversibility is denoted by the first term on the right hand side of the eq.(25) and the entropy generation due to viscous dissipation is represented by second term of eq.(25). The ratio of the entropy generation $(\mathrm{Nh})$ and the total entropy generation $(N h+N v)$ is called Bejan number $(\mathrm{Be})$. To understand the entropy generation mechanisms the Bejan number $B e$ is specified. The Bejan number for this problem can be expressed as

$$
B e=\frac{N h}{N h+N v}
$$

In general the limits of Bejan number is 0 to 1 . Finally, the irreversibility due to viscous dissipation dominant represent by $B e=0$, whereas $B e=1$ represents the domination of heat transferirreversibility on $N s$. It is clear that the heat transfer irreversibility is equal to viscous dissipation at $B e=0.5$. 


\section{VI.RESULTS AND DISCUSSION}

The effects of radiation, Joule heating, magnetic and Brinkman number on non-dimensional velocity, temperature and nanoparticle volume fraction, Bejan number $B e$ and entropy generation $N s$ are presented graphically in figures $3-7$. To study these effect of the parameters taking computations as $\mathrm{Nr}=1, \mathrm{Nb}=0.5, \mathrm{Gr}$ $=10, \operatorname{Re}=2, R=1, \operatorname{Pr}=1, A=1, L e=1, T p=0.1$.

Figure (3) displays the effect of the radiation parameter $R d$ on velocity in flow direction, temperature, nanoparticle concentration, entropy generation and Bejan number. Figure (3a) reveals that the velocity increased as enhancement in the radiation $R d$. This indicates that $R d$ have a retarding impact on the mixed convective flow. From (3b), it is noticed that $\theta(\eta)$ increased with an increment in the radiation $R d$. A raise in the radiation $R d$ parameter leads to release of heat energy in the flow direction, therefore the fluid temperature increased. Figure (3c) depicts that the nanoparticle concentration $S(\eta)$ decays with an enhancement in the radiation $R d$. Figure (3d) shows that entropy generation reduces with an enhancement in the radiation parameter $R d$. It is noticed from figure (3e) that $B e$ (Bejan number) is increasing near the lower plate of the channel, meanwhile far away from the plate the trend is reversed due to more contribution of the heat transfer irreversibility on $N s$ and $B e$ decreasing near the upper plate of the channel with enhance in $R d$.

The variation of velocity in flow direction, temperature, nanoparticle concentration, $N s$ and $B e$ with magnetic parameter $M$ is presented in figure (4). It is noticed from figure (4a) that, the dimensionless velocity decreases with an increase in the magnetic parameter $M$. Figure (4b) reveals that $\theta(\eta)$ decreases with a rise in magnetic parameter $M$. There is a raise in the nanoparticle concentration $S(\eta)$ with a rise in the magnetic parameter $M$ as depicted in figure (4c). Figure (4d) shows that entropy generation decreased with enhancement in the magnetic parameter $M$. It is clear from figure (4e) that Be is decreasing near the lower plate of the channel, meanwhile far away from the plate the trend is reversed due to more contribution of the heat transfer irreversibility on $N s$ and the $B e$ is decreasing near the upper plate of the channel with enhance in $M$.

The influence of the Brinkman number $\mathrm{Br}$ on $f(\eta), \theta(\eta), S(\eta), N s$ and $B e$ is depicted in Figure (5). The dimensionless velocity $f(\eta)$ increased with the rise in Brinkman number $B r$ as shown in figure (5a). Figure (5b) reveals that the $\theta(\eta)$ increased with a raise in the parameter $B r$. From figure (5c) it is noticed that nanoparticle concentration $\mathrm{S}(\eta)$ decay with an increment in the Brinkman number $B r$. Figure (5d) shows that the increase in the Brinkman number $\mathrm{Br}$ causes a increment in entropy generation. As increase in $\mathrm{Br}$, the Bejan number is decreasing near the lower plate of the channel, meanwhile far away from the plate the trend is reversed due to more contribution of heat transfer irreversibility on $N s$ and $B e$ increasing near the upper plate of the channel as represented in figure (5e).

The influence of the Joule heating parameter on $f(\eta), \theta(\eta), S(\eta), N s$ and $B e$ is shown in Figure (6). The velocity in flow direction increased with the rise in Joule heating parameter $J$ as shown in figure (6a). Figure (6b) explains that the $\theta(\eta)$ raises with enhancement in the Joule heating parameter $J$. From figure (6c) it is noticed that nanoparticle concentration $S(\eta)$ decreased with an enhancement in the Joule heating parameter $J$. Figure (6d) shows that the increment in Joule heating parameter $J$ raises the entropy generation. As Joule heating parameter $J$ increases, the Bejan number is decreasing near the lower plate of the channel, meanwhile far away from the plate the trend is reversed due to more contribution of heat transfer irreversibility on $N s$ and then Be increasing near the upper plate of the channel as represented in figure (6e).

Figure (7) represents the effect of thermoporesis parameter $N t$ on velocity in flow direction $f(\eta)$, nanoparticle concentration $S(\eta)$, temperature $\theta(\eta)$, Bejan number Be and entropy generation $N s$. The velocity $f(\eta)$ decreased with enhancement in the thermoporesis parameter $N t$ as shown in figure (7a). Figure (7b) reveals that, the temperature $\theta(\eta)$ increased with enhancement in thermoporesis parameter $N t$. Increase in the thermoporesis parameter $N t$ leads to increase in the effective-conductivity, hence the nanoparticle concentration $S(\eta)$ is increases as depicted in figure (7c). It is noticed from figure (7d) that as increase in the thermoporesis parameter $N t$ the entropy generation is also increased. It is seen from figure (7e) that the $B e$ is decreasing near the lower plate of the channel, meanwhile far away from the plate the trend is reversed due to more contribution of heat transfer irreversibility on $N s$ and then $B e$ is increasing near the upper plate of the channel as $N t$ increases.

\section{CONCLUSION}

In this article the laminar entropy generation in mixed convective nanofluid flow in a vertical channel has been investigated by including magnetic, Joule heating, Brinkman number and chemical reaction parameter effects. The non-dimensional non-linear equations are solved by the HAM procedure. The main observations are summarized below:

- The dimensionless velocity, temperature increased whereas the nanoparticle concentration and entropy generation decreased with raise in the thermal-radiation $R d$.

- An increase in the Brinkman number leads to increase the velocity, temperature, entropy generation and leads to decrease the nanoparticle concentration. 
- As the Joule heating parameter increases, the dimensionless temperature, velocity and entropy generation increases but the nanoparticle concentration decreases.

- The maximum values of Bejan number are observed at upper plate of the channel due to more heat transfer irreversibility on $N s$ and minimum value near the lower plate of channel due to more contribution of the fluid friction irreversibility on Ns with the increase in $R d, M, B r, J$ and $N t$.

\section{REFERENCES}

[1] Choi, S.U.S.: Enhancing thermal conductivity of fluids with nanoparticles. In Singer, D and Wang, H (Eds.), Development and Applications of Non-Newtonian Flows, American Society of Mechanical Engineers, New York, 99-106, 1995.

[2] Hang, Xu. and Pop, I. : Fully developed mixed convection flow in a vertical channel filled with nanofluids, Int. Commun. Heat Mass Transfer, vol.39, 1086-1092, 2012.

[3] Hajipour, M. and Dehkordi, A.M. : Analysis of nanofluid heat transfer in parallel plate vertical channels partially filled with porous medium, Int. J. Thermal Sciences, vol.55, 103-113, 2012.

[4] Grosan, T. and Pop, I. : Fully developed mixed convection in a vertical channel filled by a nanofluid, J. Heat Transfer, vol.134(8), 082$501,2012$.

[5] Hang, X., Tao, F. and Pop, I. : Analysis of mixed convection flow of a nanofluid in a vertical channel with the Buongiorno mathematical model, International Communications in Heat Mass Transfer, vol.44, pp. 15-22, 2013.

[6] Nield, D.A. and Kuznetsov, A.V. : Forced convection in a parallel -plate channel occupied by a nanofluid or a porous medium saturated by a nanofluid, Int. J. Heat Mass Transfer, vol.70, 430-433, 2014.

[7] Fakour, M., Vahabzadeh, A. and Ganji, D.D. : Scrutiny of mixed convection flow of a nanofluid in a vertical channel, Case Studies in Thermal Engineering vol.4, 15-23, 2014.

[8] Makhatar, N.A.M., Habibis, S. and Ishak, H. : Flow reversal of fully developed mixed convection of nanofluids in a vertical channel filled with porous medium, International Journal of Pure and Applied Mathematics, vol.103(1), 81-97, 2015.

[9] Das, S., Jana, R.N. and Makinde, O.D. : Mixed convective magneto-hydrodynamic flow in a vertical channel filled with nanofluids, Engineering Science and Technology, an International Journal, vol.18, 244-255, 2015.

[10] Chamkha, A.J., Abbasbandy, S., Rashad, A.M. and Vajravelu, K. : Radiation effects on mixed convection about a cone embedded in a porous medium filled with a nanofluid, Meccanica vol.48(2), 275-285, 2013.

[11] Sheikholeslami, M. and Ganji, D.D. : Unsteady nanofluid flow and heat transfer in presence of magnetic field considering thermal radiation, J Braz. Soc. Mech. Sci. Eng., vol.37, 895-902, 2015.

[12] Anand, R. J., Vasumathi, G. and Mounica, J. : Joule Heating and Thermal Radiation Effects on MHD Boundary Layer Flow of a Nanofluid over an Exponentially Stretching Sheet in a Porous Medium, World Journal of Mechanics, vol.5, 151-164, 2015.

[13] Srinivasacharya, D. and Vijaykumar, P. : Mixed Convection over an Inclined Wavy Surface in a Nanofluid Saturated Non-Darcy Porous Medium with Radiation Effect, International Journal of Chemical Engineering , 2015.

[14] Hayat T., Waqas M., Shehzad, S.A. and Alsaedi A. : A model of solar radiation and Joule heating in magnetohydrodynamic(MHD) convective flow of thixotropic nanofluid, Journal of Molecular Liquids, vol.215, 704-710, 2016.

[15] Bejan, A., "Second-law analysis in heat transfer and thermal design", lemph \{Adv. Heat Transfer\}, Vol. 15, pp.1 - 58, 1982.

[16] Bejan, A., "Entropy Generation Minimization", New York: CRC Press. Boca Raton.1995.

[17] Omid, M., Ali, K., Clement, K., Al-Nimr, M.A., Ioan, P., Ahmet, Z.S. and Somchai, W. : A review of entropy generation in nanofluid flow, International Journal of Heat and Mass Transfer, vol.65, 514-532, 2013.

[18] Dehsara, M. Nemat, D.and Mohammad, R.H.N. : Numerical analysis of entropy generation in nanofluid flow over a transparent plate in porous medium in presence of solar radiation, viscous dissipation and variable magnetic field, Journal of Mechanical Science and Technology, vol.28(5), 1819-1831, 2014.

[19] Govindaraju M., Vishnuganesh N., Ganga B. and Hakeem A.K. : Entropy generation analysis of magnetohydrodynamic flow of a nanofluid over a stretching sheet, Journal of the Egyptian Mathematical Society, vol.23, 429-434, 2015.

[20] Rashidi, M.M., Bhatti,M.M., Munawwar, A.A. and Mohamed, E.S.A. : Entropy Generation on MHD Blood Flow of Nanofluid Due to Peristaltic waves, Entropy, vol.18, 2016.

[21] Liao S.J. : Beyond perturbation. Introduction to homotopy analysis method. Boca Raton: Chapman and Hall/CRC Press, 2003.

[22] Sparrow E M and Cess, RD., 1978, Radiation Heat Transfer, Washington, DC: Hemisphere.

[23] Buongiorno, J. : Convective transport in nanofluids, ASME J Heat Transfer, vol.128, 240 - 250, 2006

[24] Liao S.J. : On the homotopy analysis method for nonlinear problems. Appl Math Comput., vol.147(2), 499--513, 2004.

[25] Liao S.J. : An optimal homotopy-analysis approach for strongly nonlinear differential equations. Commun Nonlinear Sci Numer Simul 15:2003-16, 2010.

[26] Liao, S.J. : Advances in the Homotopy Analysis Method. World Scientific Publishing Company, Singapore, 2013. 


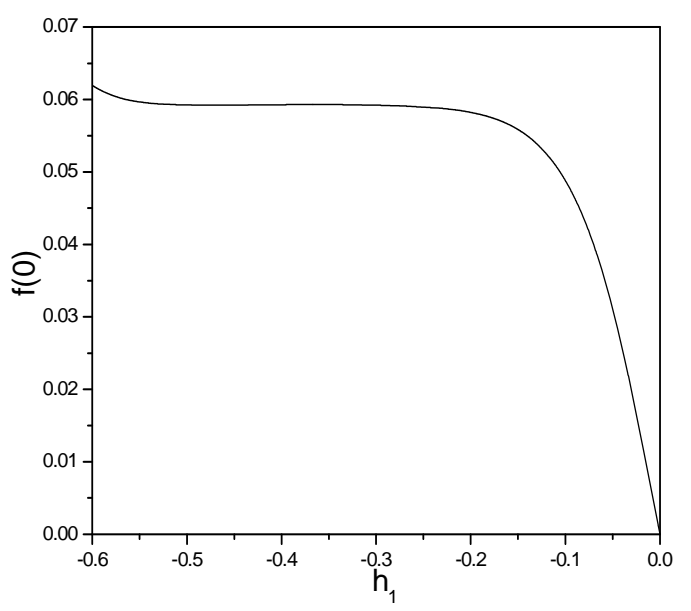

(a) $\mathrm{f}(\eta)$

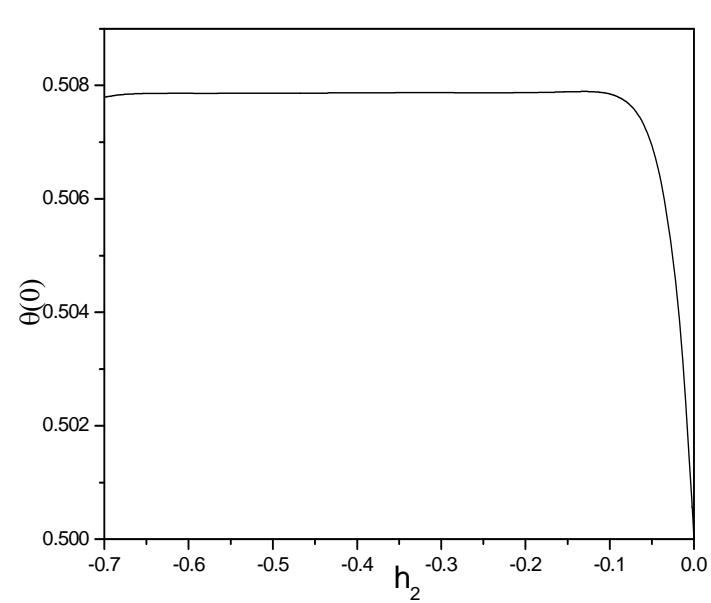

(b) $\theta(\eta)$

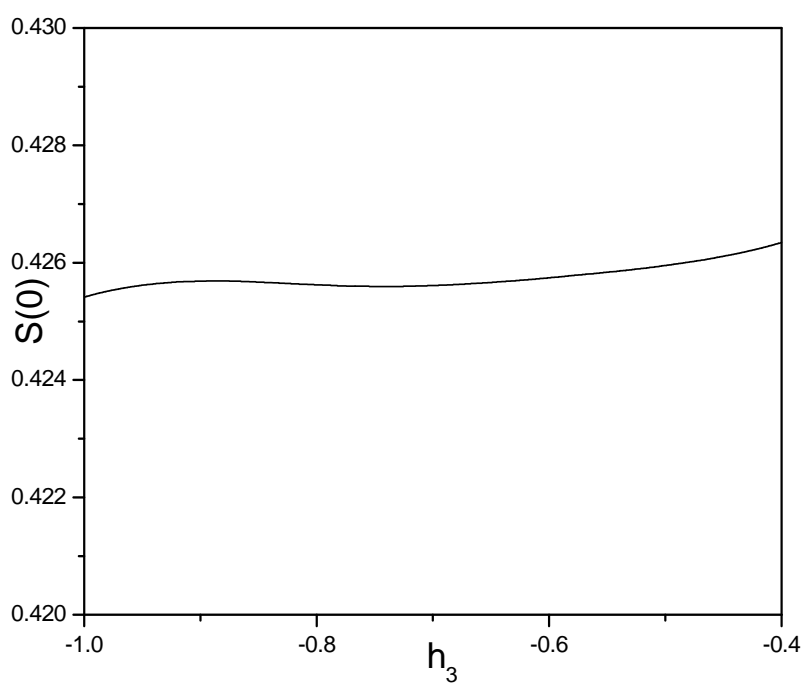

(c) $\mathrm{S}(\eta)$

Figure 2: The h-curves for $\mathrm{f}(\eta), \theta(\eta), \mathrm{S}(\eta)$ when $N r=1, N t=1, G r=10, R=1, N b=0.5, P r=1, A=1, R e=2, L e=1, R d=1.5, M=2, B r=0.5, J=2$, $T p=0.1$. 
ISSN (Print) : :2319-8613

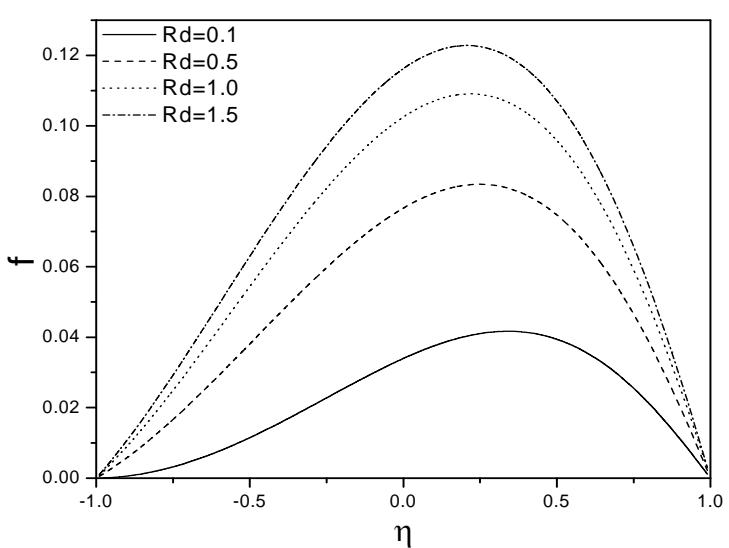

(a) $f(\eta)$

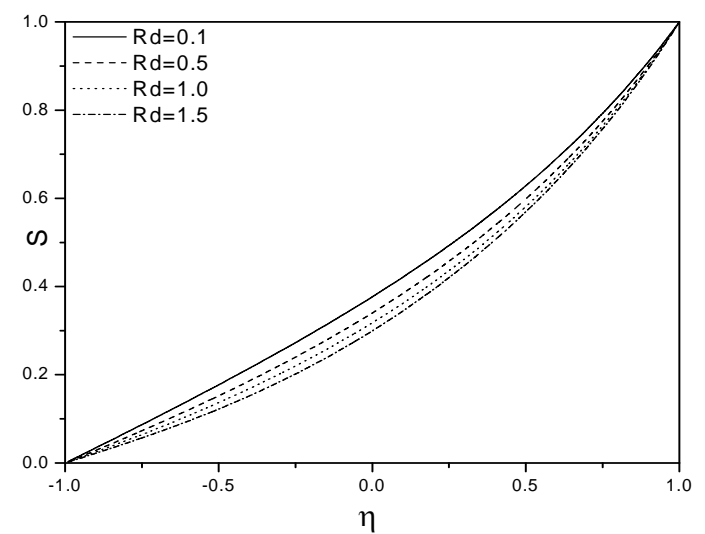

(c) $S(\eta)$

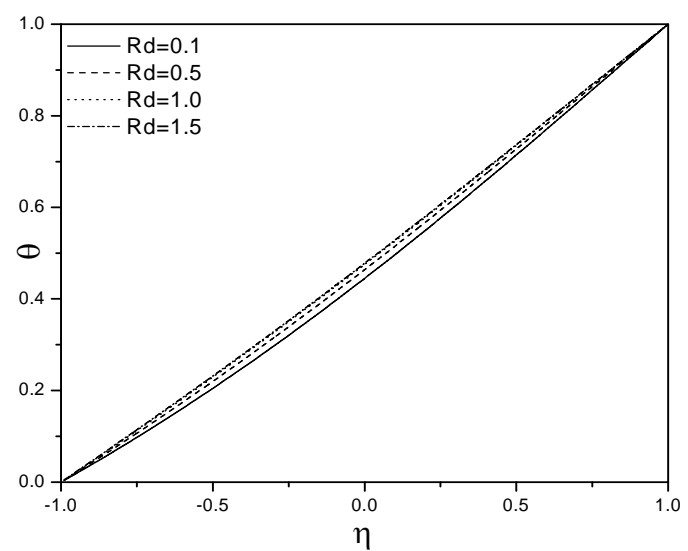

(b) $\theta(\eta)$

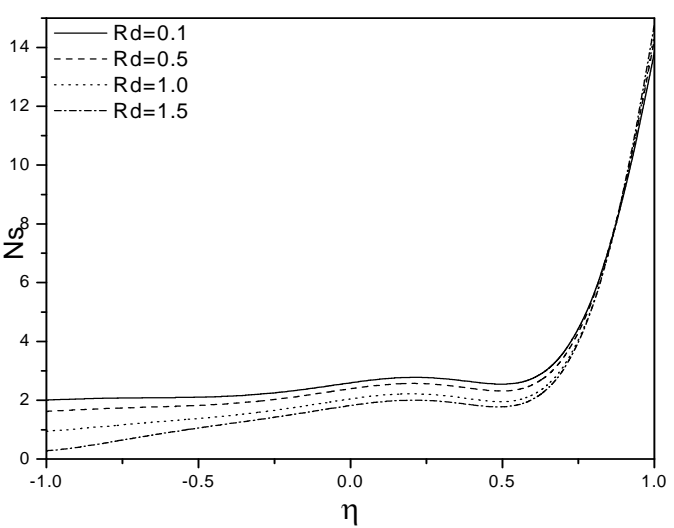

(d) $N s$

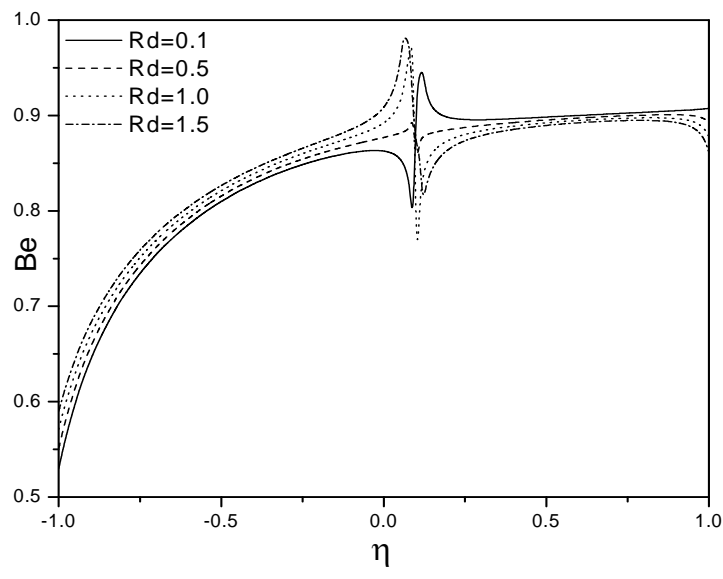

(e) $\mathrm{Be}$

Figure 3: Effect of Radiation parameter on velocity $\mathrm{f}(\eta)$, temperature $\theta(\eta)$, nanoparticle concentration $\mathrm{S}(\eta)$, Entropy generation $N s$ and Bejan number $B e$. 


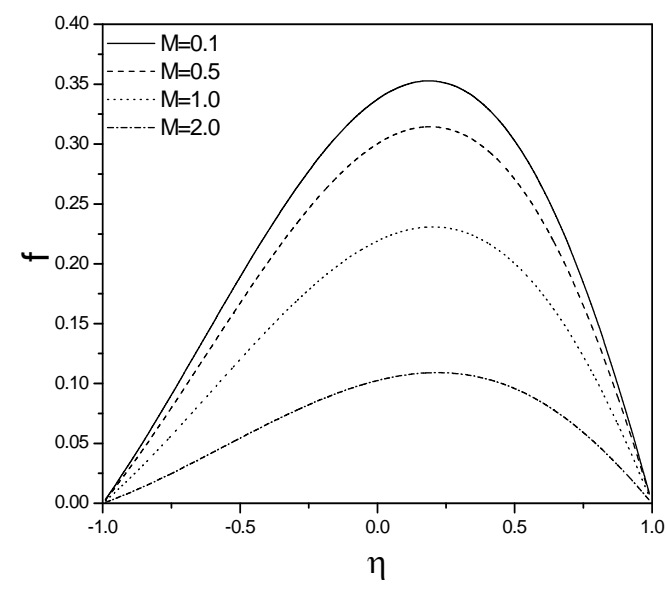

(a) $f(\eta)$

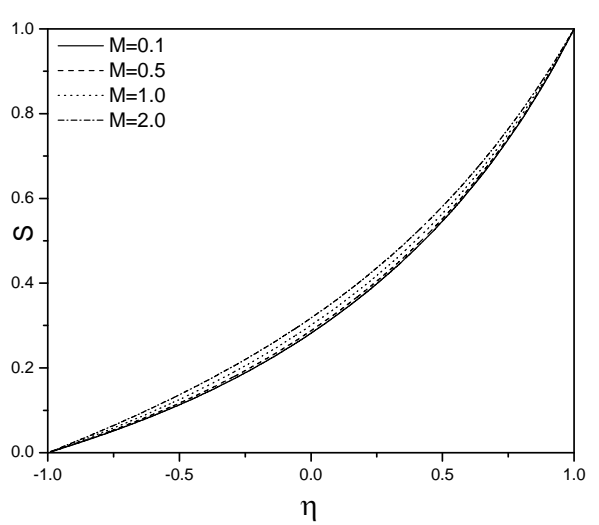

(c) $S(\eta)$

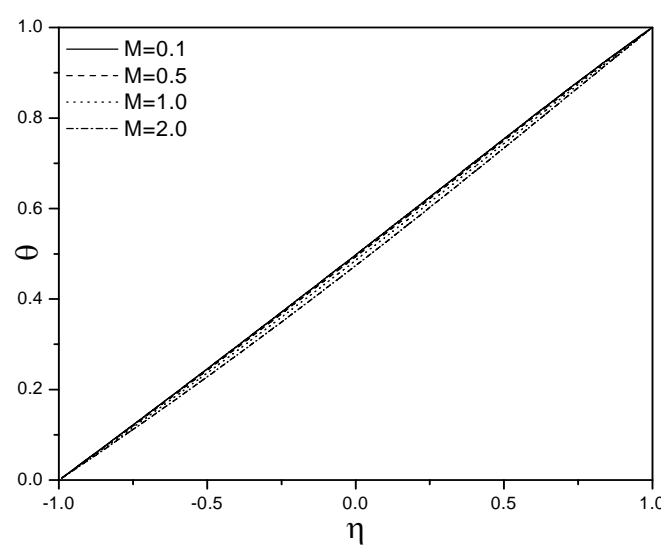

(b) $\theta(\eta)$

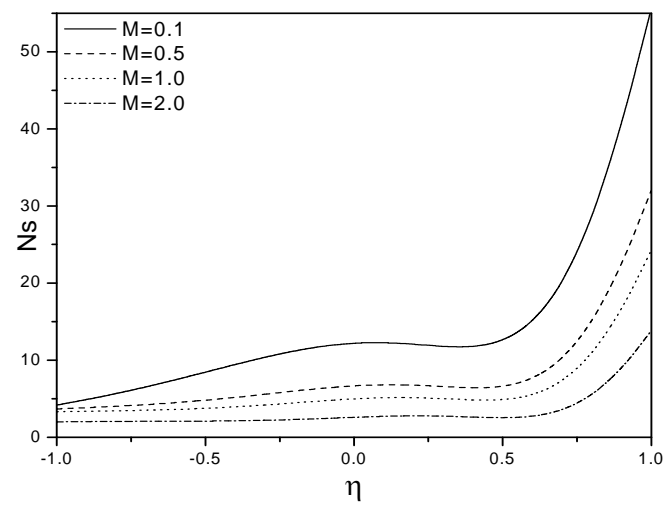

(d) $N s$

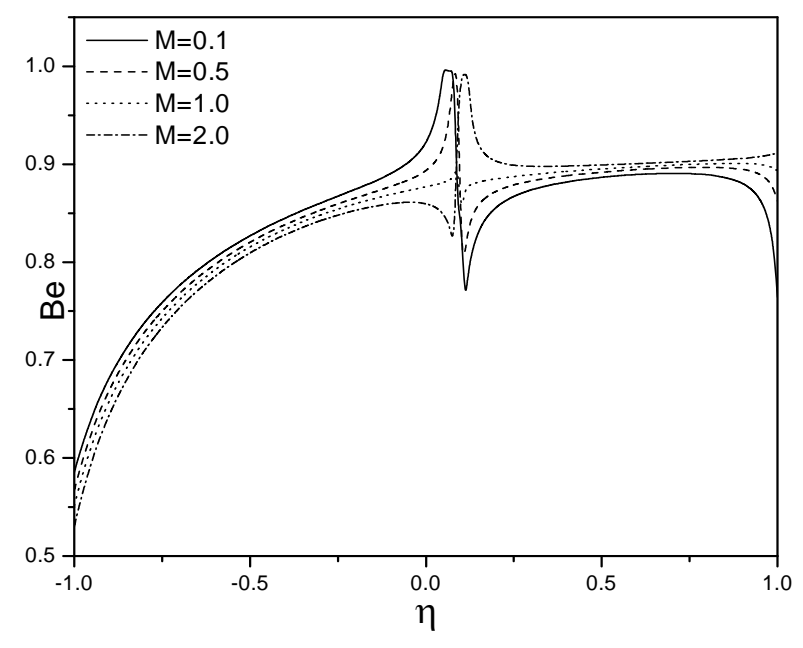

(e) $B e$

Figure 4: Effect of Magnetic parameter on velocity $\mathrm{f}(\eta)$, temperature $\theta(\eta)$, nanoparticle concentration $\mathrm{S}(\eta)$, Entropy generation $N s$ and Bejan number $B e$. 


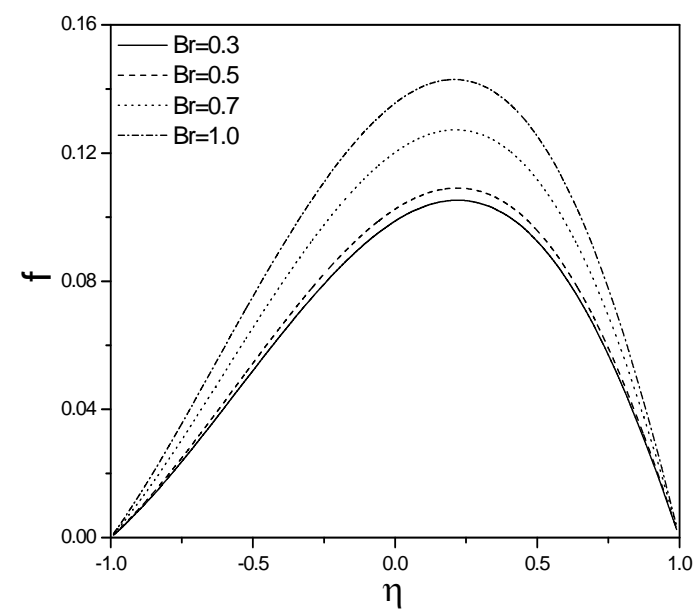

(a) $f(\eta)$

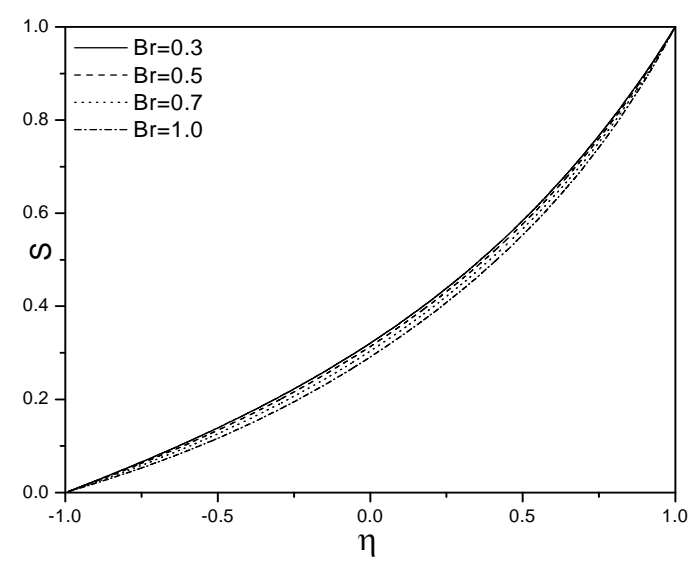

(c) $S(\eta)$

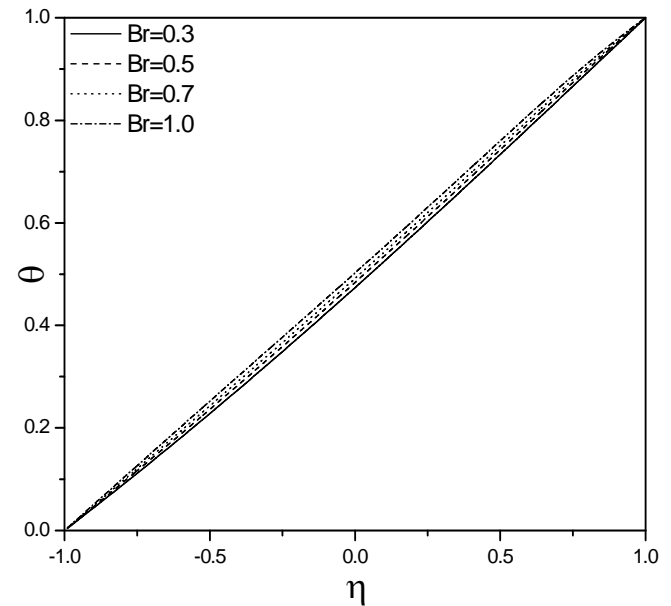

(b) $\theta(\eta)$

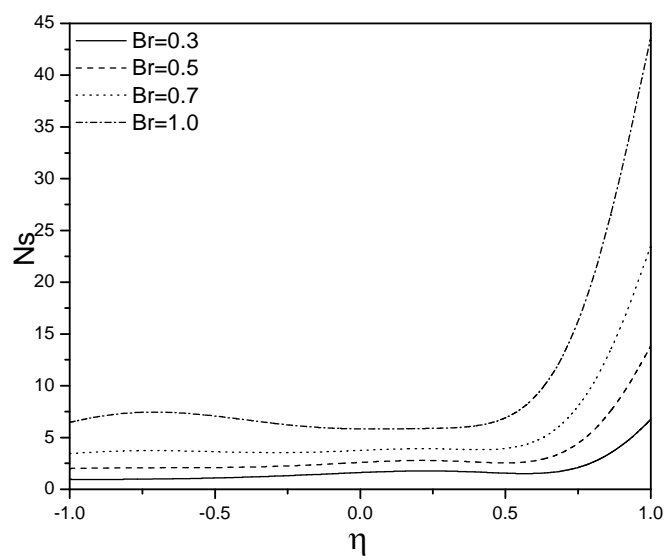

(d) $N s$

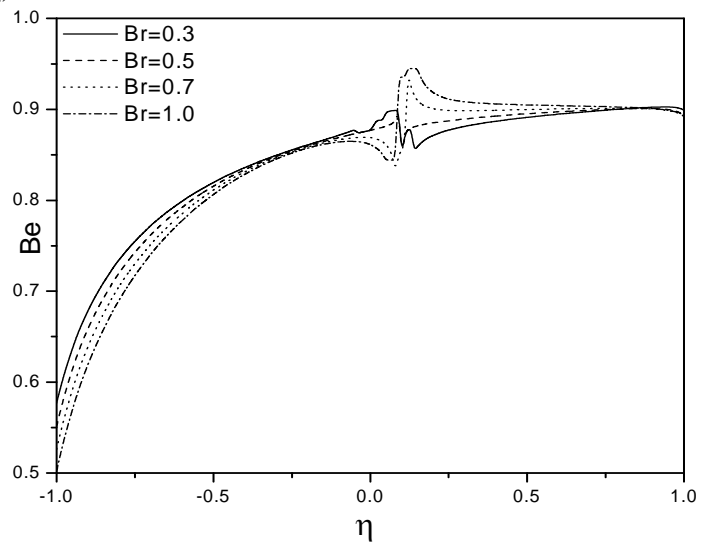

(e) $B e$

Figure 5: Effect of Brinkman number on velocity $\mathrm{f}(\eta)$, temperature $\theta(\eta)$, nanoparticle concentration $\mathrm{S}(\eta)$, Entropy generation $N s$ and Bejan number $B e$. 


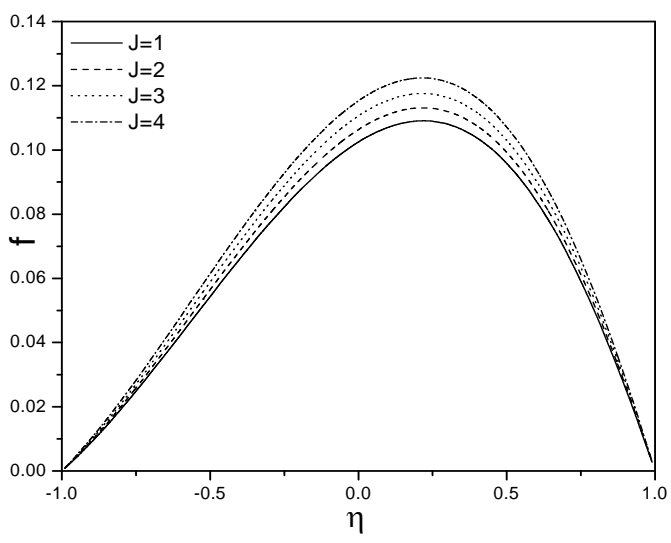

(a) $f(\eta)$

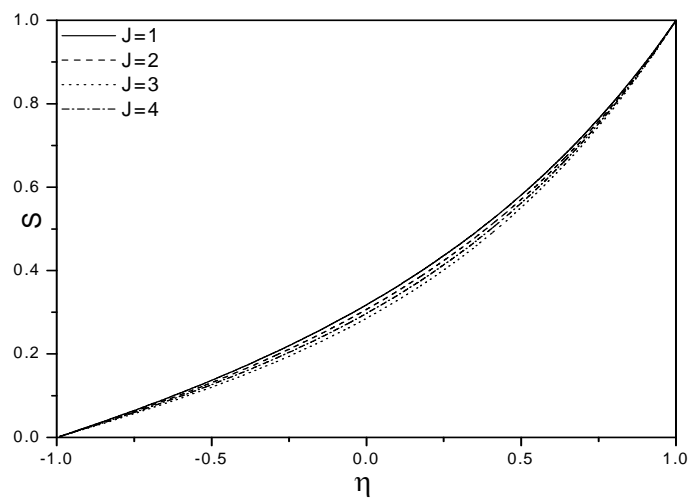

(c) $S(\eta)$

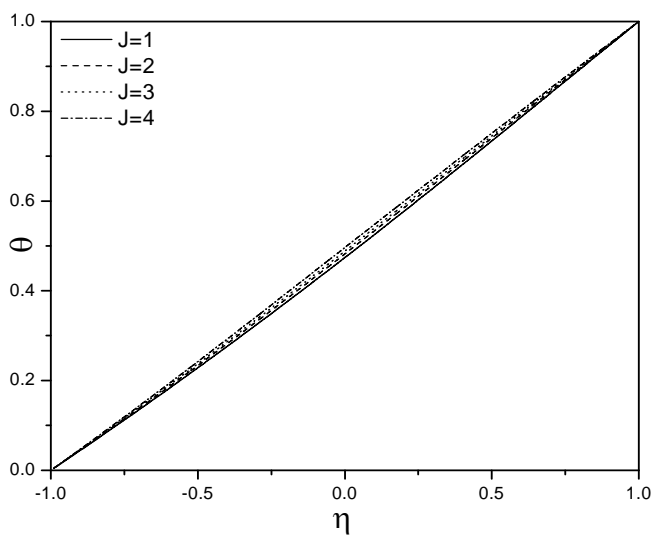

(b) $\theta(\eta)$

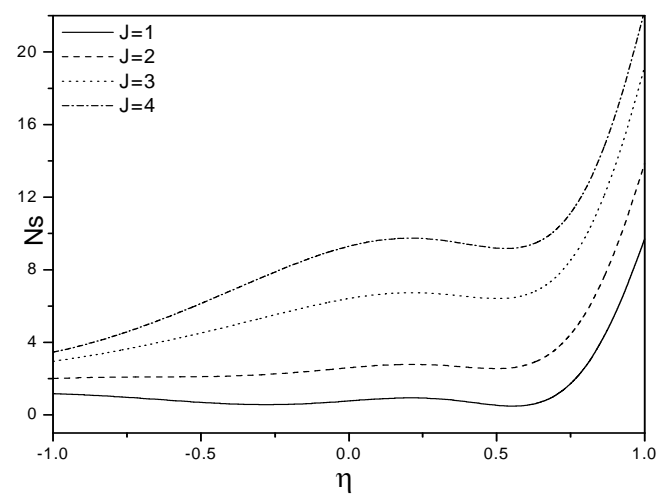

(d) $N s$

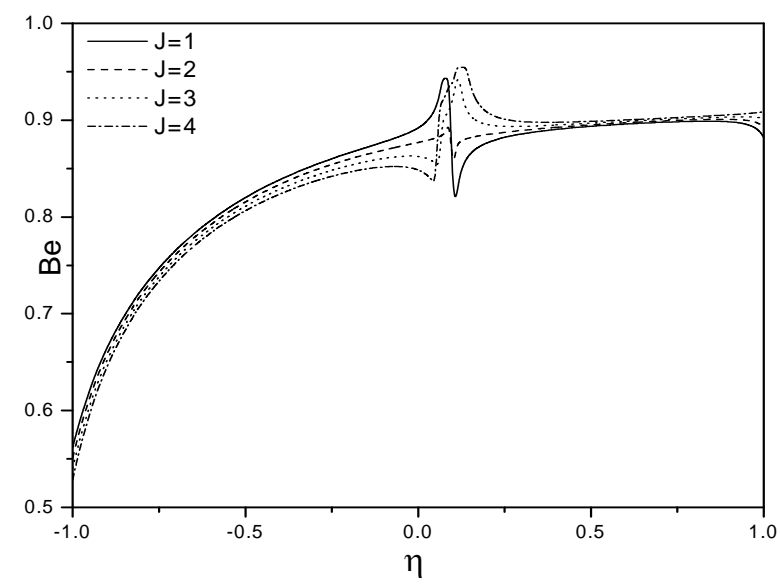

(e) $\mathrm{Be}$

Figure 6: Effect of Joule heating parameter on velocity $\mathrm{f}(\eta)$, temperature $\theta(\eta)$, nanoparticle concentration $S(\eta)$, Entropy generation $N s$ and Bejan number $B e$. 


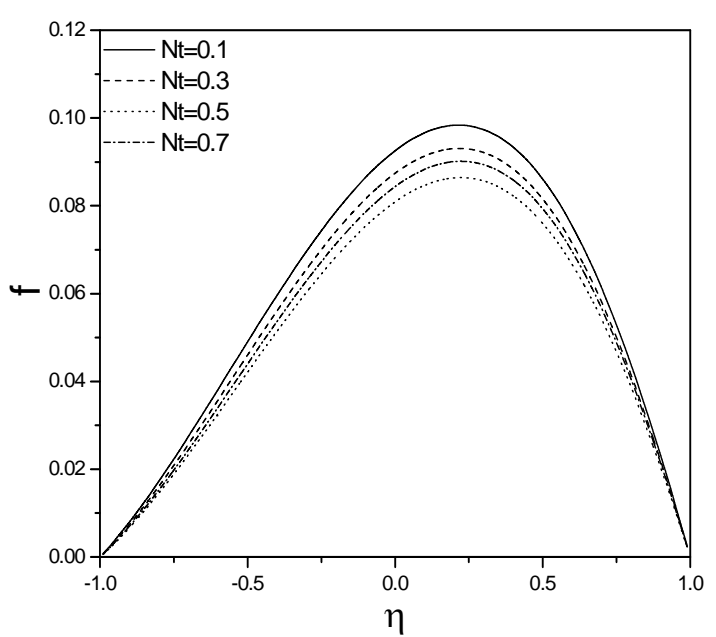

(a) $f(\eta)$

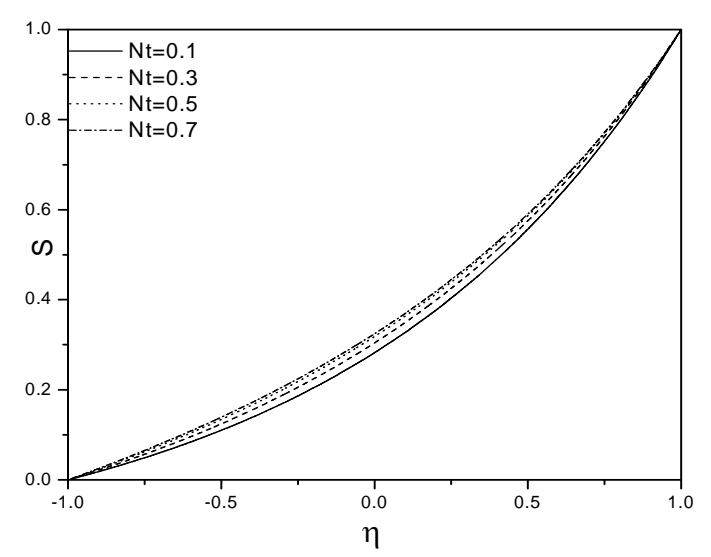

(c) $S(\eta)$

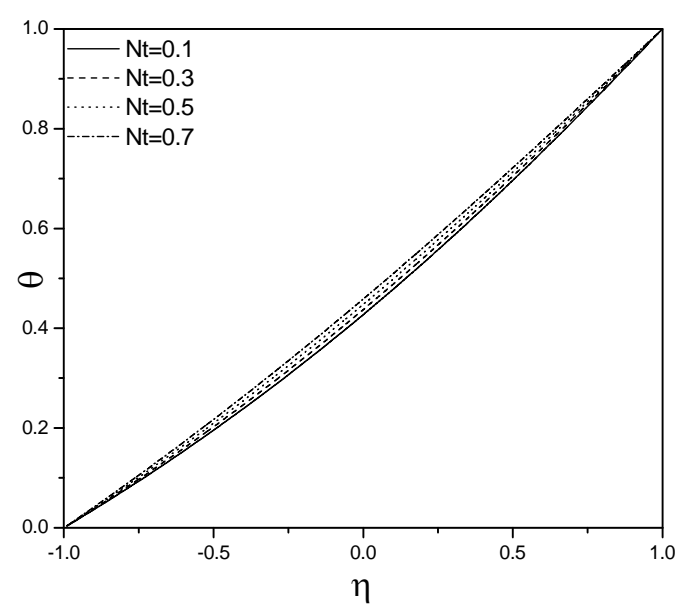

(b) $\theta(\eta)$

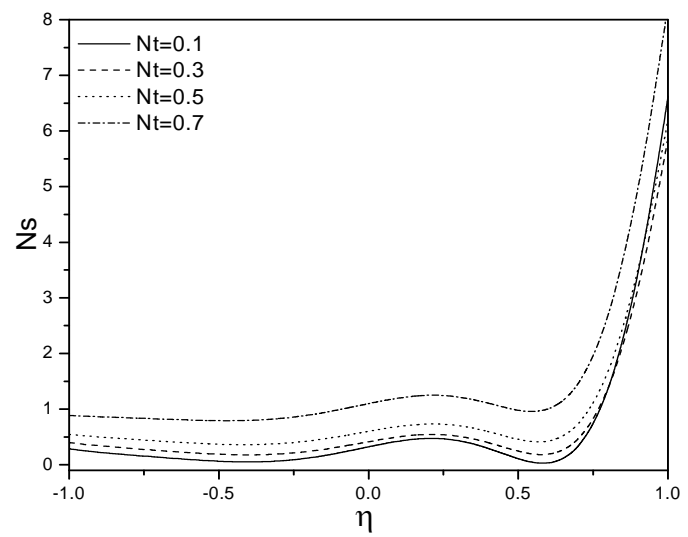

(d) $N s$

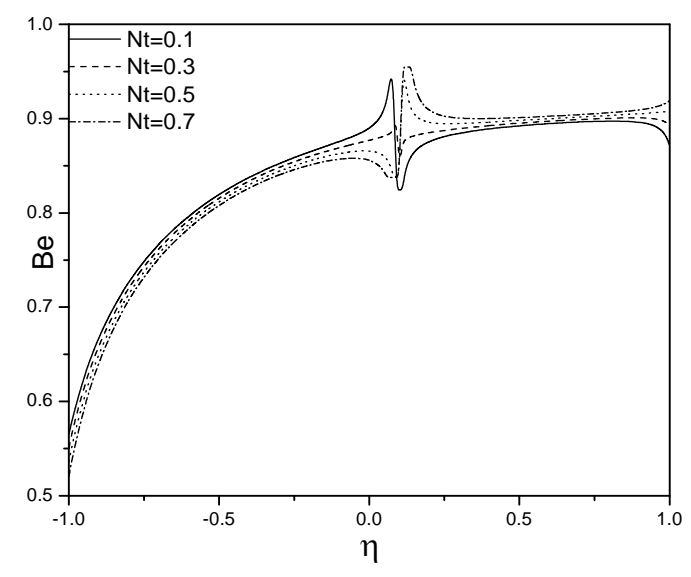

(e) $\mathrm{Be}$

Figure 7: Effect of Thermoporesis parameter on velocity $f(\eta)$, temperature $\theta(\eta)$, nanoparticle concentration $\mathrm{S}(\eta)$, Entropy generation $N s$ and Bejan number $B e$. 